\title{
Parkinson's disease as a Working Model for Global Healthcare Restructuration: The Internet of Things and Wearables Technologies*
}

\author{
C. F. Pasluosta, B. M. Eskofier \\ Digital Sports Group, Pattern Recognition Lab, \\ Department of Computer Science, Friedrich Alexander \\ University (FAU) Erlangen-Nürnberg, Erlangen, Germany \\ cristian.pasluosta@fau.de; bjoern.eskofier@fau.de
}

\author{
H. Gassner, J. Winkler, J. Klucken \\ Department of Molecular Neurology, University Hospital \\ Erlangen, FAU Erlangen-Nürnberg, Erlangen, Germany \\ heiko.gassner@uk-erlangen.de; juergen.winkler@uk- \\ erlangen.de; jochen.klucken@uk-erlangen.de
}

\begin{abstract}
With the rapid growth and increased life expectancy of the world's population, the prevalence of chronic disorders such as Parkinson's disease (PD) is also increasing. This challenges the current healthcare system in terms of cost and resources reallocation. An emerging approach facilitated by a new technological platform, called the Internet of Things, and the introduction of wearable devices for medical applications is a potential solution to this problem. Miniaturized and low-cost sensor units are placed close to the patients' body to unobtrusively collect information about their health status. This information is distributed through a smart Internet grid, creating an efficient structure that reduces costs and maximizes the resources. Patient engagement plays a key role in this healthcare restructuration. Here, we discuss the state-of-the-art of this technological revolution with emphasis in Parkinson's disease. As an example for the application of this platform to $\mathrm{PD}$, we present a potential approach to assess an important symptom- namely postural instability- using wearable sensor technology.
\end{abstract}

\section{Categories and Subject Descriptors}

H.1.2 [Models and Principles]: User/Machine Systems - Human information processing.

\section{General Terms}

Algorithms, Measurement, Experimentation, Human Factors.

\section{Keywords}

Internet of Things, wearables, Parkinson, postural instability

\section{INTRODUCTION}

As the world population continues to grow and the life expectancy to increase [1], chronic diseases of the elderly such as Parkinson's disease becomes increasingly prevalent. Our current healthcare system is challenged to meet the rising demands of patients' needs with high quality standards of care, effective applicability, and simple accessibility.

The Internet of Things (IoT) is opening the doors to new ways of optimizing resources and increasing efficiency in many fields, including home automation, agriculture and healthcare [4]. The key of its success is a lateral distribution in the production and distribution of goods and services enabled by a smart energy grid and a strong network of collaboration. This platform may provide the means to meet current expectations in healthcare delivery.

In parallel, the introduction of wearable technologies as personal objects of daily living surveys the patient health status unobtrusively in any scenario and at a very low cost. Connecting wearable devices to the IoT platform may potentiate the intrinsic features of both technologies (i.e., lateralization, connectivity, continuous monitoring, and resources optimization), shifting the paradigm of healthcare delivery.

Parkinson's disease (PD) is a progressive, chronic, and neurodegenerative disorder mostly prevalent in older adults. Its symptoms vary over the course of the disease and often within the course of the day [5]. Impairments include motor symptoms such as bradykinesia, tremor, rigidity and postural instability [6]. Other non-motor symptoms include sleep disorders, dementia, and cognitive decline. Thus, the management of Parkinson's disease using wearable technologies and the IoT is an excellent working model of this new integrated technological platform.

Here, we discuss the technological aspects involving PD, wearable technologies and the IoT platform, accompanied by the protagonistic role of patients in this high-tech developing process. We also present preliminary results on the estimation of postural instability in PD patients as a working example of the potential of this new technology.

\section{A PARADIGM SHIFT IN PARKINSON'S DISEASE}

\subsection{The Internet of Things and Wearable Technologies}

The IoT concept relies on connecting any physical object to the Internet [7]. The number of objects connected to the Internet has rapidly increased in the past decade with 50 billion devices with ${ }^{*}$ Internet connection predicted by 2020 [8].

The IoT platform rests mainly on three concepts: (1) Renewable energy, to sustainably power up the technological platform; (2) Connectivity, to ensure the efficient flow of information; and (3) Collaboration, as the only way to improve efficiency through a lateral distribution of information, goods, and services [9]. This

\footnotetext{
* Part of this work was submitted to the IEEE JBHI [2] and the 37th Ann. Int. Conf. of the IEEE EMBS [3].
} 
also includes the arising of a new type of consumers that create, share, and consume their own products and services [9].

Along with this technological phenomenon, the accelerated development in the last decade of miniaturized, low-cost and efficient sensor units allowed the design of wearable and unobtrusive devices [10]. These sensor units are not only capable of sensing motion and physiological data, but also of performing low level of signal and data processing. Moreover, the arising of 3D printing technology may take the design and production of wearable sensors a step forward as these sensors might in the future be designed and fabricated at home, with some examples already in their research stage [11], [12].

The emerging of wearable technologies enhances the connectivity achieved through the IoT platform [13]. Mobile devices gather spatial and temporal information of both the individual and the community, enabling society based large-scale phenomena screening and mapping [14].

\subsection{New Ways of Knowledge Discovery}

Wearable technologies embedded into the IoT platform present a unique opportunity to generate data with valuable information to the research and medical community. However, collecting unbiased data is only the first stage in generating real benefits to the patients. Further processing is required to transform these data into knowledge, which can be used to design and implement novel diagnostics and treatments.

With motor impairment being one of the cardinal symptoms of Parkinson's disease, analyzing gait patterns to gather information regarding the stage of the disease is an intuitive approach. In this direction, wearable motion sensors attached to the patient shoes presents as an inexpensive and valuable option [15]-[18].

Gait and other body motion analysis using wearable technology are applicable to several fields. Instrumented medical examinations such as the timed up and go (TUG) test and posturography assessments can for example complement traditional medical examinations [19]-[21]. Individualized invasive procedures such as deep brain stimulation can be also optimized using information from wearable technologies [22]. Further, the assessment of new medication and rehabilitation therapies can be improved by wearable sensors [23].

Interconnecting wearable devices, ubiquitous devices embedded in the surrounding space and other objects of daily living (e.g., home appliances and ambient sensors) through the IoT may capture novel information and hence knowledge regarding the patient's health, which would otherwise be impossible to uncover during regular medical visits. This information can be delivered back to the users and their caregivers in the way of unobtrusive displays, and vibrotactile and audible cues. This will complement the efforts of physicians and other caregivers to deliver high quality of care at an affordable cost.

\subsection{Patient Engagement}

The involvement of patients in the diagnosis and treatment of their own disease will transform the way that Parkinson's disease and other chronic disorders are managed. Patients share their experience and medical information freely in the Internet [24], providing new venues for caregivers to interact with patients and for scientists to explore new research directions.

Novel approaches of data sharing impact patient-to-patient interactions as well as the research and medical community as this information can be used for assessment of new treatment and medications. Moreover, self-reported data can be utilized to further investigate new medications in large, non-controlled, open label, and observational study designs, which are initially examined in small cohorts using best-evidence clinical study design [25]. This in turn may bring controversies (and a complete new way of quality control in data generation), but shows a clear path where patient engagement may dramatically change treatment design.

The extension of this concept to automatic data generation using wearable sensors and distributed through the IoT platform is not far from attainment. Increasing patient engagement through userfriendly technology will reshape the way Parkinson's disease is managed, with individualized diagnosis and treatment concepts.

\section{POSTURAL INSTABILITY: AN EXAMPLE IN PARKINSON'S DISEASE}

Postural instability is one of the cardinal symptoms of Parkinson's disease and its clinical examination is an important part of the diagnostic workup. The most common clinical method to quantify postural instability in PD is the Pull Test performed by the doctor [26], which is included in the Unified Parkinson's Disease Rating Scale (UPDRS). It consists of pulling the patient backwards from his or her shoulders and rate how the patient stabilize from this postural perturbation. The physician's (semi-quantitative and nonlinear) ratings of the outcome are 0 (normal), 1 (retropulsion with unassisted recovery), 2 (the patient falls if without assistance), 3 (tendency to spontaneous imbalance), and 4 (test not possible, the patient requires assistance to stand) [26]. This examination aims at obtaining surrogate information on the patient's performance during the activities of daily live, in particular predicting gait alterations leading to increased risk of falling.

In the current medical settings, patients are required to visit a clinical institution to obtain an assessment of their postural instability. This imposes costs on patient transportation, the clinician's consultation and the hospital infrastructure. An automatic and at-home procedure for postural instability assessment would reduce these costs significantly. Further, the subjective nature of the test makes the outcomes dependent on the clinician's expertise and opinion [27]. This introduced bias may be also minimized with an automatic and objective instrumented test setting, or other sensor-based information on gait instability.

Wearable technology in combination with the IoT platform presents as a unique solution to this problem. In research, wearable sensor placed at the patient's trunk and lower back have been used to measure postural instability in PD as new instrumented tools, or tests that correlated with accepted scores [20]-[21], [28]-[29]. However, gait and foot motion analysis using wearable sensors remains poorly investigated. Placing unobtrusive sensors to record patient's foot motion patterns to infer postural stability is an intuitive approach for measuring postural instability, which can be easily implemented at home. This could potentially automate the diagnosis, improving efficiency and patient satisfaction.

\subsection{Estimating Postural Instability}

As a first step towards developing an unbiased and automated approach to assess postural instability, we proposed estimating the Pull Test outcomes using the information from gait sequences and foot-motion tasks.

We studied 139 idiopathic PD patients (phenotypes: bradykinesia, tremor-dominant and equivalent) equipped with a commercial inertial sensor unit in each shoe as part of a sensor-based analysis 
system (eGaIT, Astrum IT GmbH, Erlangen, Germany). The patients performed two gait tasks including walking 40 meters (40MW) and walking 20 meters with stops (SG). Patients also performed two foot-motion tasks, including circular movements of the foot (CL) and heel-to-toe tapping (HTT). Previous to these tasks, a physician performed the Pull Test for each participant.

From these tasks, we recorded 3-D acceleration and 3-D orientation data from which we extracted and selected features to classify the Pull Test scores (Figure 1). We utilized machine learning algorithms (i.e., Support vector machine as the classifier with a radial basis function as the kernel) to classify the patient score. We included patients with a Pull Test score of 0,1 and 2 . We further investigated which task produces better classification results for each patient phenotype.

A cross-validation assessment using a probabilistic approach [30] revealed that we were able to estimate the Pull Test scores from gait and foot-motion derived data similarly as the physician's ratings, with an overall accuracy of $75 \%$ (Table 1 ).

Table 1. Classification performance results.

\begin{tabular}{|l|l|l|c|}
\hline \multicolumn{1}{|c|}{ Data set } & $\mathbf{N}^{\circ}$ of Features & \multicolumn{1}{c|}{ Mean } & $\mathbf{9 5 \%}$ CI \\
\hline Complete & 413 & 0.75 & {$[0.69-0.82]$} \\
\hline Equivalent (CL) & 38 & 0.79 & {$[0.69-0.87]$} \\
\hline Bradykinetic (CL) & 57 & 0.75 & {$[0.64-0.85]$} \\
\hline Tremor-dominat (SG) & 38 & 0.70 & {$[0.56-0.83]$} \\
\hline
\end{tabular}

\subsection{Future Work}

The preliminary yet promising value of the presented methodology warrants further evaluation on the use of wearable sensors attached to the patient's shoes. This includes the implementation of an objective assessment such as posturography. Analyzing the relationships between postural sway during posturography measurements and gait could be potentially used in at-home assessments of postural instability.

Further work also includes designing practical and user-friendly protocols for patients to perform at home. Physician follow-up via telemedicine may complement the assessment.

\section{FUTURE CHALLENGES}

\subsection{Data Management}

Data handling is one of the major challenges in data-driven knowledge discovery. Avoiding information overload requires an efficient bidirectional flow of multidimensional data, which requires smarter and more robust data architecture. Many barriers regarding data sharing, data privacy and ownership must be overcome prior to adoption of this technology by the general society [31]-[33]. This discussion is currently under ethical, social and legal debates [34].

\subsection{Smarter technologies}

Home monitoring is a primary goal in the applicability of wearable technologies and the IoT to Parkinson's disease. However, its implementation is still under development. Progress in this direction is hampered by a deficit of robust and flexible technologies capable of translating lab-based results into unconstrained scenarios. Further, the large inter- and intra-subject variability of symptoms in PD patients presents as another obstacle in this scenario.

Machine-machine interaction remains also underdeveloped, necessitating more robust designs and implementations. Currently, multi-level and different sensor modalities do not interact with

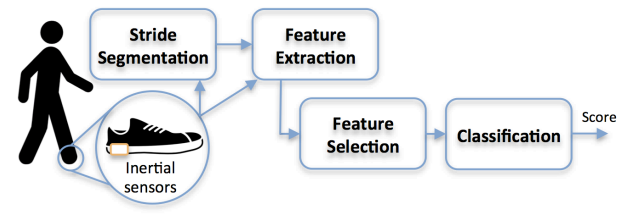

Figure 1. Block diagram of the classification pipeline.

each other (e.g., ambient sensors do not communicate with bodyworn sensors). The potential benefits of combined machinemachine interaction with the IoT platform include fall prevention and other interventions, which otherwise would be impossible to achieve without human supervision.

\subsection{Collaborations}

Cooperation is the core of the IoT platform and a fundamental piece of the lateralization of the new healthcare system. Hardware and software require designs towards integration of information, facilitating collaborative efforts. This demands open access frameworks and sharing benchmarking platforms. Human expertise within multidisciplinary teams is critical to integrate not only the research and medical community, but also patients and family members. Even though this process has begun, there is still much work to do toward an integrative management in Parkinson's disease.

\section{CONLUSIONS}

A new approach to healthcare delivery in Parkinson's disease is emerging to respond to the intense demands and needs of a larger and older elderly population. Wearable technology and the new technological platform of the Internet of Things are shaping this transition from a centralized structure to a more lateraleddistributed healthcare architecture. Engaging patients in the management of their own disease represents a key aspect of this transition.

\section{ACKNOWLEDGMENTS}

This study is supported by the FAU Emerging Fields Initiative (EFIMoves, 2_Med_03). The graphics in this article were in part generated from graphic resources designed by Freepik.com.

\section{REFERENCES}

[1] United Nations, Department of Economic and Social Affairs, Population Division (2013). World Population Prospects: The 2012 Revision, Volume I: Comprehensive Tables ST/ESA/SER.A/336.

[2] C. Pasluosta, H. Gassner, J. Winkler, J. Klucken, and E. Bjoern M., "An Emerging Era in the Management of Parkinson's disease: Wearable Technologies and the Internet of Things," IEEE J. Biomed. Heal. Informatics, (in Press).

[3] C. Pasluosta, J. Barth, H. Gassner, J. Klucken, and E. Bjoern M., "Pull Test Estimation in Parkinson's Disease Patients using Wearable Sensor Technology," in 37th Ann. Int. Conf. of the IEEE EMBS, Milan, 2015.

[4] T. McCourt, S. Leopold, F. Louthan, H. Mosesmann, S. Smigie, T. Tilman, D. Toomey, G. Kyriakopoulos, E. Lemus, B. Peterson, and A. Sklar, "The Internet of Things: A study in hype, reality, disruption, and growth," Raymond James \& Associates, USA, 2014.

[5] C. a Davie, "A review of Parkinson's disease.," Br. Med. Bull., vol. 86, pp. 109-27, Jan. 2008. 
[6] J. Jankovic, "Parkinson's disease: clinical features and diagnosis.," J. Neurol. Neurosurg. Psychiatry, vol. 79, no. 4, pp. 368-76, Apr. 2008.

[7] A. Gabbai. (2015, Jan.). "Kevin Ashton describes 'the Internet of Things': The innovator weighs in on what human life will be like a century from now. Smithsonian Magazine, USA. [Online]. Available:

http://www.smithsonianmag.com/innovation/kevin-ashtondescribes- the-internet-of-things-180953749/?no-ist.

[8] D. Evans, "The Internet of Things-How the next evolution of the internet is changing everything," Cisco, USA, 2011.

[9] Rifkin, The Zero Marginal Cost Society: The Internet of Things, the Collaborative Commons, and the Eclipse of Capitalism. New York, NY, USA: Palgrave Macmillan Trade, 2014.

[10] X. Teng, Y. Zhang, C. C. Y. Poon, P. Bonato, and S. Member, "Wearable Medical Systems for p-Health," IEEE Rev. Biomed. Eng., vol. 1, pp. 62-74, 2008.

[11] V. Lakafosis, A. Rida, R. Vyas, L. Yang, S. Nikolaou, and M. M. Tentzeris, "Progress towards the first wireless sensor networks consisting of inkjet-printed, paper-based RFIDenabled sensor tags," Proc. IEEE, vol. 98, no. 9, pp. 16011609,2010

[12] S. B. Kesner and R. D. Howe, "Design principles for rapid prototyp- ing forces sensors using 3D printing," IEEE ASME Trans. Mechatron., vol. 16, no. 5, pp. 866-870, Oct. 2011.

[13] S. Hiremath, G. Yang, and K. Mankodiya, "Wearable Internet of Things: Concept, Architectural Components and Promises for Person-Centered Healthcare," Proc. 4th Int. Conf. Wirel. Mob. Commun. Healthc., pp. 304-307, 2014.

[14] R. Ganti, F. Ye, and H. Lei, "Mobile crowdsensing: current state and future challenges," IEEE Commun. Mag., vol. 49, no. November, pp. 32-39, 2011.

[15] S. R. Hundza, W. R. Hook, L. Member, C. R. Harris, S. V Mahajan, P. A. Leslie, C. A. Spani, L. G. Spalteholz, B. J. Birch, D. T. Commandeur, and N. J. Livingston, "Accurate and Reliable Gait Cycle Detection in Parkinson's Disease," IEEE Trans. Nerural Syst. Rehabil. Eng., vol. 22, no. 1, pp. 127-137, 2014.

[16] J. Barth, J. Klucken, P. Kugler, T. Kammerer, R. Steidl, J. Winkler, J. Hornegger, and B. Eskofier, "Biometric and mobile gait analysis for early diagnosis and therapy monitoring in Parkinson's disease.," Proc. 33rd Annu. Int. Conf. IEEE Eng. Med. Biol. Soc., pp. 868-71, 2011.

[17] J. Klucken, J. Barth, P. Kugler, J. Schlachetzki, T. Henze, F. Marxreiter, Z. Kohl, R. Steidl, J. Hornegger, B. Eskofier, and J. Winkler, "Unbiased and mobile gait analysis detects motor impairment in Parkinson's disease.," PLoS One, vol. 8, no. 2, p. e56956, Jan. 2013.

[18] A. Rampp, J. Barth, S. Schülein, K.-G. Gaßmann, J. Klucken, and B. M. Eskofier, "Inertial sensor based stride parameter calculation from gait sequences in geriatric patients," IEEE Trans. Biomed. Eng., vol. 62, no. 4, pp. 1089-1097, 2015.

[19] C. Zampieri, A. Salarian, P. Carlson-Kuhta, K. Aminian, J. G. Nutt, and F. B. Horak, "The instrumented timed up and go test: potential outcome measure for disease modifying therapies in Parkinson's disease.," J. Neurol. Neurosurg. Psychiatry, vol. 81, no. 2, pp. 171-6, Feb. 2010.
[20] L. Palmerini, L. Rocchi, S. Mellone, F. Valzania, and L. Chiari, "Feature selection for accelerometer-based posture analysis in Parkinsons disease," IEEE Trans. Inf. Technol. Biomed., vol. 15, no. 3, pp. 481-490, 2011.

[21] M. Mancini, A. Salarian, P. Carlson-Kuhta, C. Zampieri, L. King, L. Chiari, and F. B. Horak, "ISway: a sensitive, valid and reliable measure of postural control," J. Neuroeng. Rehabil., vol. 9, no. 1, p. 59, 2012.

[22] S. Patel, C. Mancinelli, R. Hughes, A. Dalton, L. Shih, and P. Bonato, "Optimizing deep brain stimulation settings using wearable sensing technology," 2009 4th Int. IEEE/EMBS Conf. Neural Eng., pp. 6-9, Apr. 2009.

[23] S. Patel, H. Park, P. Bonato, L. Chan, and M. Rodgers, "A review of wearable sensors and systems with application in rehabilitation.," J. Neuroeng. Rehabil., vol. 9, no. 1, p. 21, Jan. 2012.

[24] J. Heywood, Benjamin Heywood and J. Cole, "PatientsLikeMe," 2005. [Online]. Available: http://www.patientslikeme.com/. [Accessed: 09-Apr-2015].

[25] P. Wicks, T. E. Vaughan, M. P. Massagli, and J. Heywood, "Accelerated clinical discovery using self-reported patient data collected online and a patient-matching algorithm.," Nat. Biotechnol., vol. 29, no. 5, pp. 411-4, May 2011.

[26] A. L. Hunt and K. D. Sethi, "The pull test: a history.," Mov. Disord., vol. 21, no. 7, pp. 894-9, Jul. 2006.

[27] R. P. Munhoz, J.-Y. Li, M. Kurtinecz, P. Piboolnurak, a. Constantino, S. Fahn, and a. E. Lang, "Evaluation of the pull test technique in assessing postural instability in Parkinson's disease," Neurology, vol. 62, no. 1, pp. 125-127, Jan. 2004.

[28] E. Grimpampi, V. Bonnet, A. Taviani, and C. Mazzà, "Estimate of lower trunk angles in pathological gaits using gyroscope data," Gait Posture, vol. 38, no. 3, pp. 523-527, 2013.

[29] W. Maetzler, M. Mancini, I. Liepelt-Scarfone, K. Müller, C. Becker, R. C. van Lummel, E. Ainsworth, M. Hobert, J. Streffer, D. Berg, and L. Chiari, "Impaired trunk stability in individuals at high risk for Parkinson's disease," PLoS One, vol. 7, no. 3, 2012.

[30] H. Carrillo and K. H. Brodersen, "Probabilistic performance evaluation for multiclass classification using the posterior balanced accuracy," in ROBOT2013: First Iberian Robotics Conference, 2013, pp. 347-361.

[31] W. G. van Panhuis, P. Paul, C. Emerson, J. Grefenstette, R. Wilder, A. J. Herbst, D. Heymann, and D. S. Burke, “A systematic review of barriers to data sharing in public health," BMC Public Health, vol. 14, no. 1, p. 1144, 2014.

[32] A. Mashhadi, F. Kawsar, and U. G. Acer, "Human Data Interaction in IoT: The ownership aspect," Internet Things (WF-IoT), 2014 IEEE World Forum, pp. 159-162, 2014.

[33] E. Stuart, M. Moh, and T.-S. Moh, "Privacy and security in biomedical applications of wireless sensor networks," in Proc. 2008 First Int. Symp. Appl. Sci. Biomed. Commun. Technol., pp. 1-5, Oct. 2008.

[34] Manzeschke, K. Weber, E. Rother, and H. Fangerau, "Ethical ques- tions in the area of age appropriate assisting systems," German Federal Ministry of Education and Research, Munich, 2015. 